\title{
Localization of benign Insulinomas using Glucagon-like Peptide-1 Receptor (GLP-1R) SPECT/CT and PET/CT in a prospective clinical study
}

Kwadwo Antwi ${ }^{1}$, Melpomeni Fani ${ }^{1}$, Tobias Heye ${ }^{1}$, Guillaume Nicolas ${ }^{1}$, Elmar Merkle ${ }^{1}$, Jean Claude Reubi ${ }^{2}$, Beat Gloor ${ }^{3}$, Emanuel Christ ${ }^{\star} 4$, Damian Wild $^{\star 1}$ * = shared last authors

${ }^{1}$ Clinic of Radiology and Nuclear Medicine, University of Basel Hospital, Switzerland

2Division of Experimental Pathology, Department of Pathology, University of Bern, Switzerland

${ }^{3}$ Department of Visceral Surgery, University Hospital of Bern, Switzerland

${ }^{4}$ Division of Diabetology, Endocrinology, and Metabolism, Inselspital Bern, University Hospital and University of Bern, Switzerland

\section{Background}

- In patients with benign insulinomas conventional imaging is able to detect about $60-70 \%$ of the small pancreatic lesions $(1-2 \mathrm{~cm})$.

- Surgery is the only curative option for this disease.

- Preoperative localisation of the tumour is critical for the surgical strategy.

- Benign insulinoma express in nearly $100 \%$ GLP-1 receptors at a high density (ca. $5 x$ higher than in normal beta-cells).

- GLP-1R single Photon Emission Computed Tomography (SPECT) has been shown to be a valid non-invasive tool for the localisation of benign insulinoma.

- A prospective randomized study comparing GLP-1R Positron Emission Tomography (PET)/CT and GLP-1R SPECT/CT has not yet been performed.

\section{Aim}

To compare the detection rate of GLP-1R PET/CT and GLP-1R SPECT/CT in patients with a biochemically proven endogenous hyperinsulinemic hypoglycemia.

\section{Methods}

- Adult patients with with neurogylcopenic symptoms due to endogenous hyperinsulinemic hypoglycemia were enrolled (ClinicalTrials.gov: NCT02127541).

- No signs of malignancy on conventional imaging.

- Investigations included ${ }^{111} \mathrm{In}-\mathrm{DOTA}$-exendin-4 SPECT/CT and ${ }^{68} \mathrm{Ga}-$ DOTA-exendin-4 PET/CT in a randomized order.

- Endpoint was correct detection rate (gold standard: histology).

\section{Results}

-Thirty-three patients (25 females, 8 males, age range 18-80 years, mean 49 years) were scanned until now.

-Previously performed cross-sectional imaging (CT/MRI) was negative or not conclusive in $25 / 33(76 \%)$ of patients.

-22 patients have been operated, two patients refused surgery and five patients are awaiting surgery.

- In this collective, the histopathological diagnosis of a benign insulinoma was confirmed in 19 patients, 1 patient had an adult islet cell hyperplasia. In 1 patient both intraoperative palpation and histological diagnosis did not confirm an insulinoma. In 1 patient symptoms of endogenous hypoglycemia ceased postoperative but histological diagnosis did not confirm the diagnosis. This patient was excluded from evaluation as the final diagnosis remained unclear. In 4 patients PET/CT, SPECT/CT as well as the previous performed conventional imaging did not find any suspicious lesion and were not operated up to date.

In this interim analysis:

$>$ PET/CT (2.5h p.i.) showed an overall pooled sensitivity of $95 \%$

SPECT/CT (72h p.i.) showed an overall pooled sensitivity of $73 \%$ -PET/CT was the only modality which correctly identified the area of islet cell hyperplasia (adult nesidioblastosis) within the pancreas.

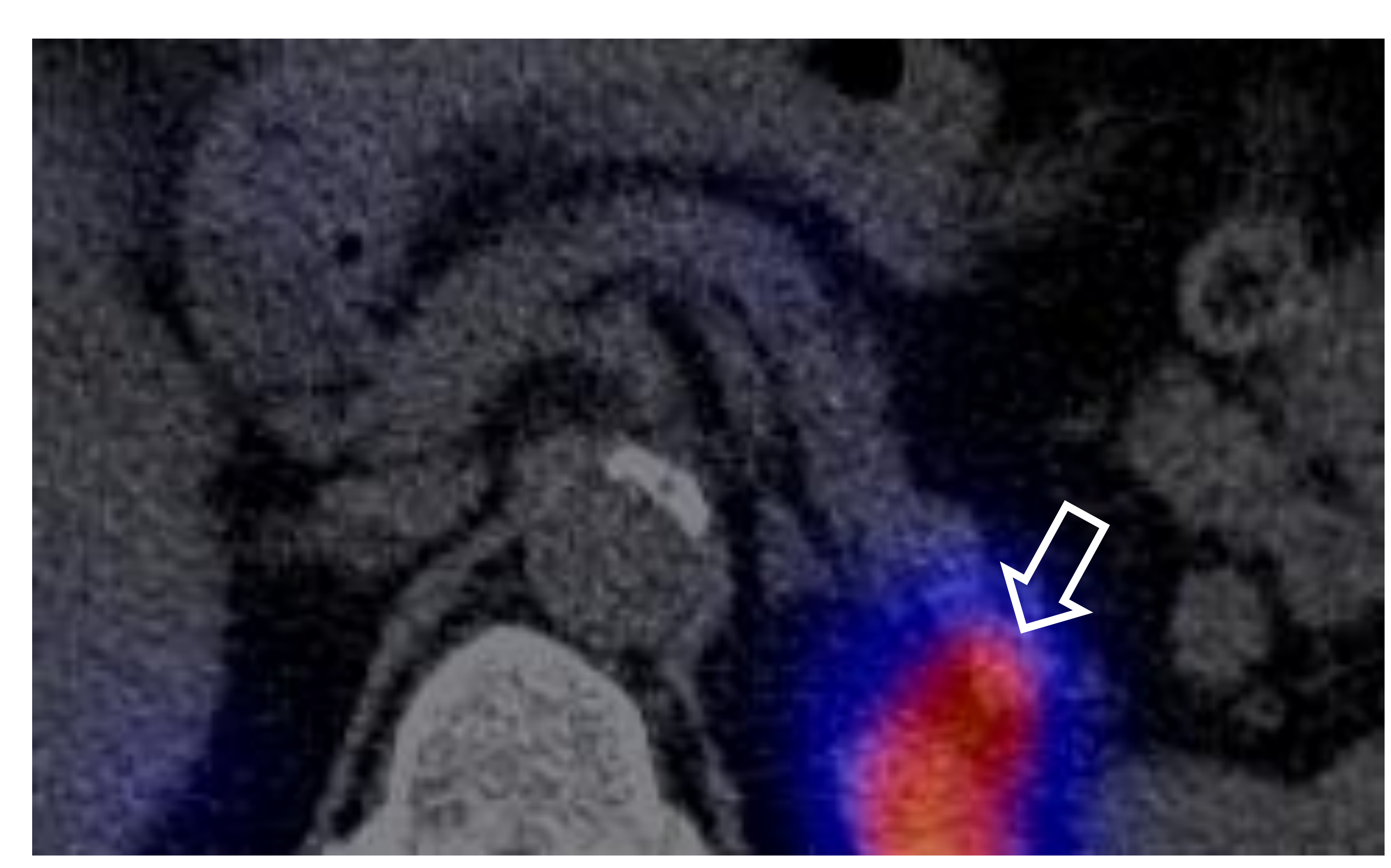

Figure 1: Transaxial ${ }^{111} \mathrm{In}$-DOTA-exendin-4 SPECT/CT. The arrow shows focal uptake in the distal portion of the pancreatic tail.

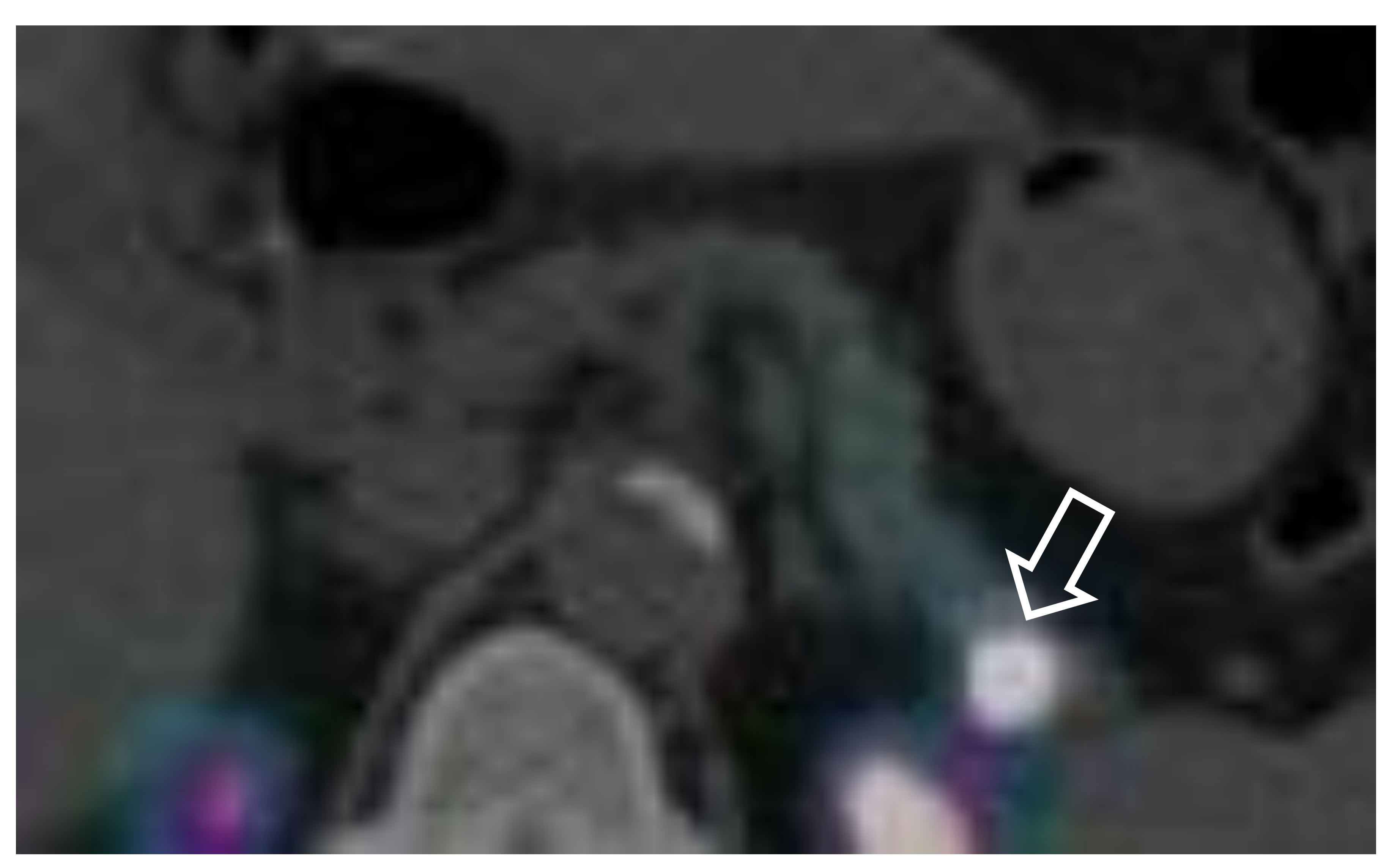

Figure 2: Transaxial ${ }^{68} \mathrm{Ga}-\mathrm{DOTA}-\mathrm{exendin}-4 \mathrm{PET} / \mathrm{CT}$. The arrow shows focal uptake in the distal portion of the pancreatic tail (same patient).

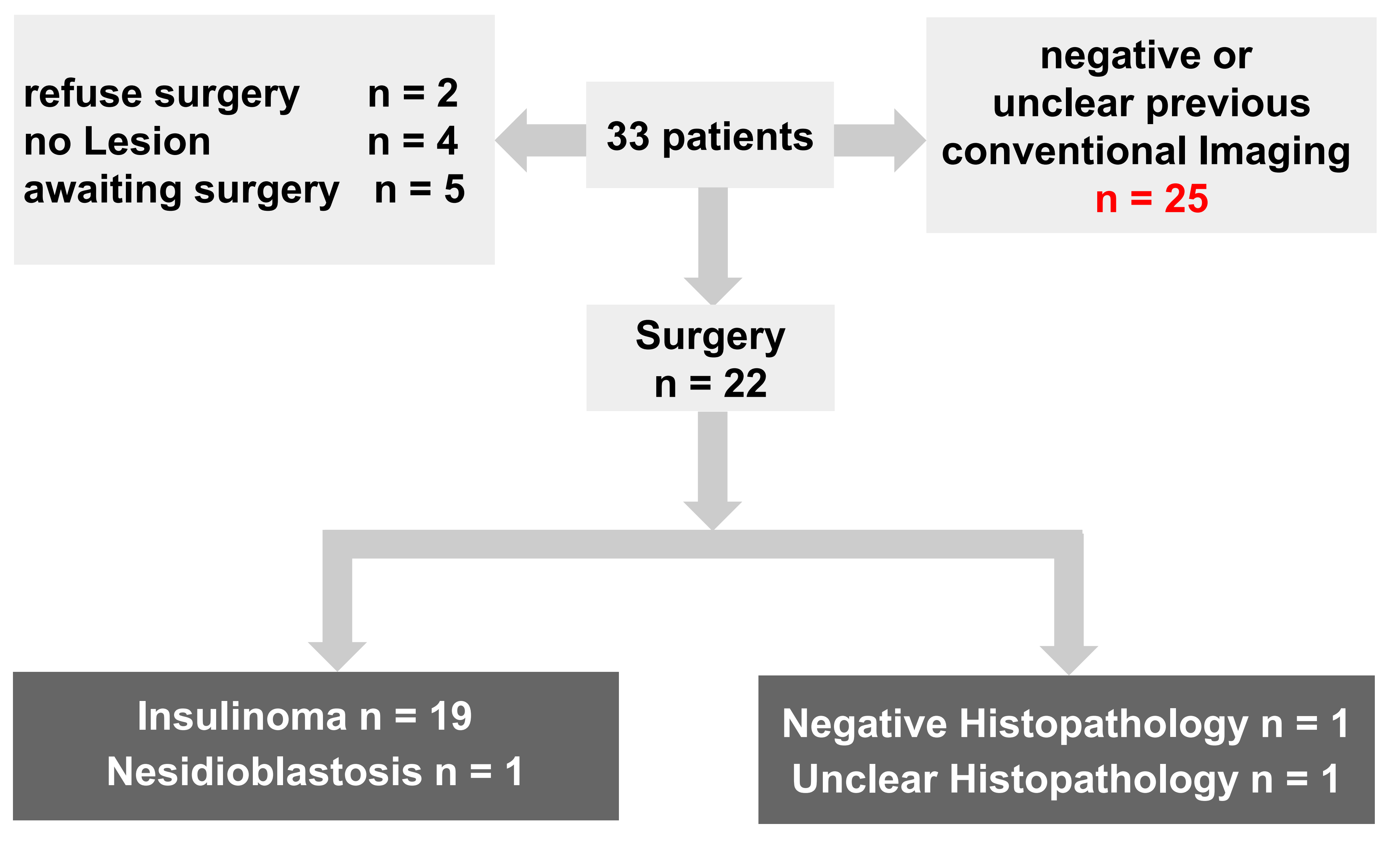

Figure 3: Results

\section{Conclusion}

1.Our interims analysis suggests that GLP-1R PET/CT performs better than GLP-1R SPECT/CT at a lower radiation dose and shorter examination time.

2.GLP-1R PET/CT will be a useful diagnostic tool in patients where cross sectional imaging (CT/MRI) fails to localize the insulinoma. 